\title{
Big Data Analysis in Healthcare
}

\author{
Seewon $\mathrm{Ryu}, \mathrm{PhD}^{1}$, Tae-Min Song, $\mathrm{PhD}^{2}$ \\ ${ }^{1}$ Inje Institute of Advanced Studies, Seoul; ${ }^{2}$ Korea Institute for Health and Social Affairs, Seoul, Korea
}

The rise of big data is promising new opportunities to understand and forecast contexts or problems in the healthcare area. Big data related to healthcare has explosively expanded with the development of the Internet. It is not only accumulated in purely healthcare service settings but also in cyber-digital space, such as traditional and mobile Internet, smartphones, smart TVs, sensor and RFID-based ubiquitous networks, and social media [1]. Information and communication technology is the main driver of voluminous and varying big data to be managed and analyzed. These big data, including text, audio, video, or any other digital medium can be analyzed to provide new and useful information.

Big data analytics has opened a new era to improve services and solve problems in the healthcare area. Big data analytics has recently attracted interest because it couples social data analytics to traditional analytics, though data analytics has long been important in science and healthcare practice. Forecasting using Google Flu Trends (GFT), beginning in February 2013, has attracted interest beyond the healthcare area, and it illustrates the new usefulness and value of social data and big data analytics. This innovative estimation of flu prevalence of GFT has opened up and motivated new possibilities of handling long-lasting problems in the healthcare area by using social data in cyber space. Many stakeholders recognize that big data analytics may provide opportunities for forecasting, discovering tentative needs, and reducing risks, as well as providing personalized services, real-time sensing and counter-measures more appropriately.

New applications of big data analytics in the healthcare area

This is an Open Access article distributed under the terms of the Creative Commons Attribution Non-Commercial License (http://creativecommons.org/licenses/bync/3.0/) which permits unrestricted non-commercial use, distribution, and reproduction in any medium, provided the original work is properly cited.

(c) 2014 The Korean Society of Medical Informatics are being developed and introduced all over the world. Seton Healthcare, working with predictive analytics, sought to address the need to reduce the occurrence of high-cost congestive heart failure readmissions [2]. The European Commission has developed 'MediSys', an information scanning tool, to reinforce the network for the surveillance of communicable diseases and the early detection of bioterrorist activities. Breaking news can be detected by algorithm of MediSys using over 20,000 Internet articles per day produced by the Europe Media Monitor and can be sent to key persons by email and SMS. Many successful big data applications are being introduced [3]. Many healthcare institutions and countries have done many trials and made many successful cases of big data analysis to solve traditional problems in healthcare, such as reducing readmissions, increasing the effectiveness and efficiency of healthcare, improving the quality of care, and forecasting demands for healthcare services.

There are several factors that must be taken into consideration in using big data analytics for healthcare, although it has the potential to provide insights to enhance our understand of changing phenomena. Big data must be prepared systematically and must be of good quality. Regarding big data analytics, we should remember the popular saying 'garbage in, garbage out'. Proper big data analytics using highly qualified big data would produce useful and valuable results for understanding contexts and forecasting the future of healthcare. Next, we have not to think and use the results of big data analytics as being one and powerful information to understand and forecast something interested. The interpretation of results must be done cautiously by experts who come from diverse fields of expertise and have a wide variety of perspectives. We have to use big data analytics cautiously even after strategic consideration, though it has much potential to improve our understanding of phenomena and strengthen our ability to accurately forecast the future. 
Finally, we think that academic study of big data analysis could contribute to and expand the practical implementation of big data analytics. Such study should focus on any meaningful and objective criteria, such as clinical and social effectiveness, efficiency, usefulness, and adoption of big data analysis.

\section{References}

1. Song TM. Efficient utilization of big data on healthcare and welfare area. Healthc Welf Forum 2012;193:68-76.

2. IBM. Reducing readmissions to improve care [Internet]. Armonk (NY): IBM; c2014 [cited at 2014 Oct 20]. Available from: http://www-01.ibm.com/software/sg/ industry/healthcare/pdf/setonCaseStudy.pdf.

3. European Commission. Medical intelligence in Europe [Internet]. Brussel: European Commission; c2014 [cited at 2014 Oct 20]. Available from: http://ec.europa.eu/ health/preparedness_response/generic_preparedness/ planning/medical_intelligence_en.htm. 